\title{
WAFER-TO-WAFER TRANSFER OF MICROSTRUCTURES FOR VACUUM PACKAGING
}

\author{
Michael B. Cohn, Yiching Liang*, Roger T. Howe, and Albert P. Pisano* \\ Departments of Electrical Engineering \& Computer Science and Mechanical Engineering* \\ Berkeley Sensor \&.Actuator Center \\ Berkeley, CA 94720-1770
}

\begin{abstract}
Vacuum packages based on wafer-to-wafer transfer of polysilicon shells are described. Transfer is accomplished by Au/ $\mathrm{Si}$ eutectic bonding, in conjunction with break-away tethers. The low bonding temperature $\left(370^{\circ} \mathrm{C}\right)$ enables end-process application. The short anneal time ( -30 seconds) and wafer-scale parallelism promise high manufacturing throughput. Inexpensive packages of this type are desired for resonant sensors and implantable devices. However, a more general application may lie in assembly of the devices themselves, i.e. by transfer of prefabricated sensor structures, circuits, and other components.
\end{abstract}

\section{INTRODUCTION}

Resonant microstructures require a vacuum environment to achieve a high mechanical Q. Examples of this sensor class include resonant accelerometers [1] and vibratory rate gyroscopes [2], [3], [4]. Volume manufacturing of these devices will most likely require a micromachined vacuum encapsulation process, which would allow the sensor chip to be enclosed in a conventional, low-cost electronic package.

Several groups have demonstrated micropackaging processes, based on surface micromachining, wafer bonding, eutectics, and other methods. The current work borrows elements from all of these, but introduces a new technique, namely the batch transfer of released microstructures from one wafer to another. In this method, micromachined shells are fabricated on a handle wafer, using a sacrificial layer process such as HexSil [5]. As shown in Figure 1, the shells are anchored to the substrate by tethers, and coated with gold. The handle wafer is then contacted to a second wafer and heated to the Au-Si eutectic point. When cool, the two wafers are separated, breaking the tethers and leaving the shells welded to the second substrate.

This transfer process achieved $100 \%$ yield in chip-level transfers, with 30 packages per chip. Vacuum packaging was achieved by annealing in vacuum. At the end of a 5-month test, substantial vacuum persisted. Precision alignment was obtained by mechanical interference fit between lithographically defined features on the two substrates. An unexpected advantage of this process is its robustness to surface roughness and particulate contamination.

\section{BACKGROUND}

Micromachined packaging dates to Wise's 1981 singlecrystal silicon hemispherical microshells for inertial fusion targets [6]. Petersen [7] has shown pressure sensors with sealed reference cavities fabricated by silicon fusion bonding. Schmidt [8] produced sealed pressure sensors by $\mathrm{Si} / \mathrm{SiO}_{2}$ wafer bonding and etchback. Ikeda [9] and Guckel [10] have demonstrated surface micromachined encapsulation for resonant beams, evacuated by reactive sealing.

Various glass packages have been shown, typically anodically bonded to millimeter-scale sensor chips or thin-film structures [11], [12].

Mastrangelo [13] and Lin [14] have demonstrated vacuum
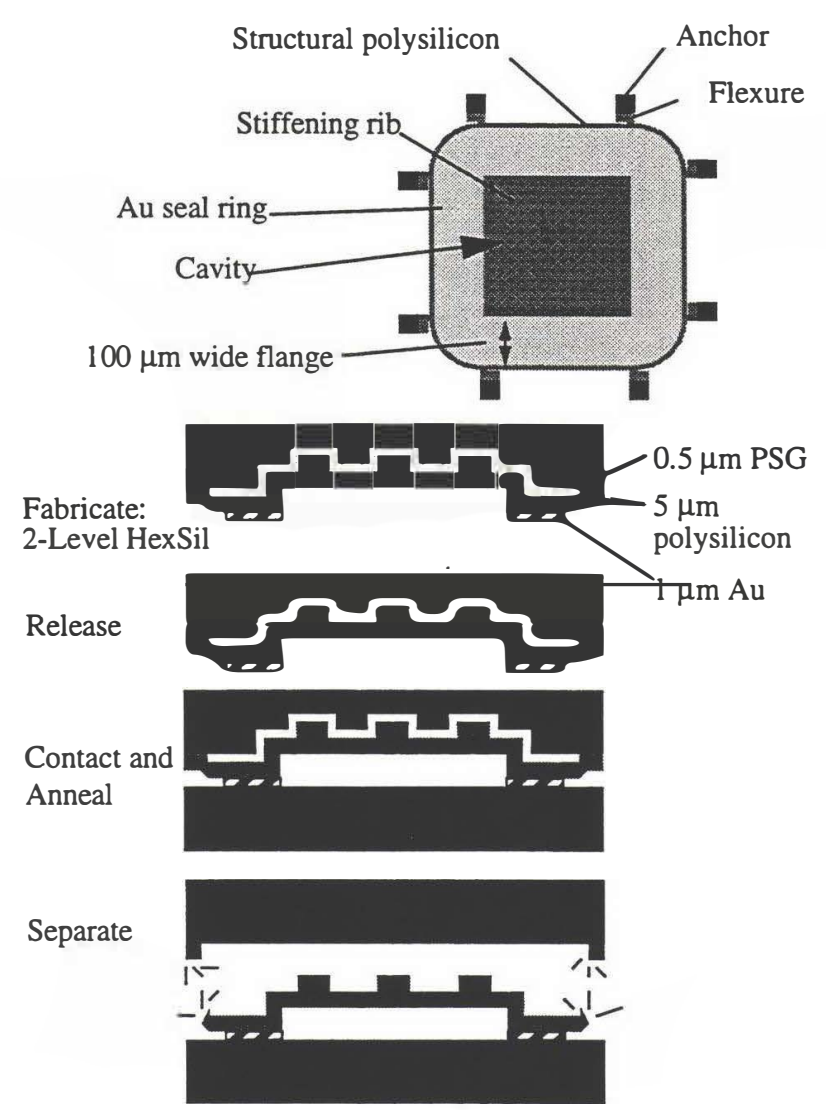

FIGURE 1. Above: Layout of a typical polysilicon cap for vacuum packaging. Below: Cross-section view of bonding process.

packages for incandescent microlamps and microresonators, respectively. In this work, a silicon nitride film was deposited over a sacrificial PSG layer; the resulting shells were etched out with HF and sealed by CVD. The final pressure is limited by residual CVD gases.

Several researchers have employed eutectic bonding, both for assembly and packaging [15], [16]. An advantage of this process is its low temperature: e.g. $183^{\circ} \mathrm{C}$ for the $\mathrm{Pb} / \mathrm{Sn}$ system, and $363^{\circ} \mathrm{C}$ for $\mathrm{Au} / \mathrm{Si}$.

\section{PROCEDURE AND RESULTS}

The basic packaging procedure consists of two parts: fabrication and transfer. The packages - essentially polysilicon hemishells - are fabricated by the HexSil process [5]. Starting with a $<100>$ silicon substrate, an anisotropic plasma etch defines a mold for the package's stiffening ribs. These have a width of $8 \mu \mathrm{m}$ and a depth of $5 \mu \mathrm{m}$, and are laid out in a cartesian grid. A second plasma etch defines cavities $300 \mu \mathrm{m}$ square and $4 \mu \mathrm{m}$ deep for the packages themselves. This depth should not be exceeded without special resist coating equipment, or step coverage problems may occur in subsequent lithography.

The etched surfaces are smoothed by a wet silicon etch 
( $50 \mathrm{HNO}_{3}: 20 \mathrm{H}_{2} \mathrm{O}: 1 \mathrm{NH}_{4} \mathrm{~F}, 5 \mathrm{~min}$ ), followed by growth of $0.5 \mu \mathrm{m}$ of wet thermal oxide, and removal of the oxide in concentrated $\mathrm{HF}$.

A sacrificial oxide is then deposited. PSG is preferred, since release of the caps will require $250 \mu \mathrm{m}$ of undercut. LTO may be used as a diffusion barrier to protect adjacent layers from doping. The film is densified at $1000^{\circ} \mathrm{C}$ for 1 hour in nitrogen.

The oxide is patterned with anchor holes. A $20 \mu \mathrm{m}$ square geometry proves adequate, though other sizes may be possible. In photoresist application, care should be taken to ensure adequate coverage around the cavities. Multiple coats may be required, particularly if plasma etching. Adhesion promoters such as HMDS may be detrimental. If a spin coater is used, "stale" photoresist must be purged from the feed line. A minimal hardbake should be used, as resist tends to pull back from steps.

Polysilicon deposition is preceded by a 15 second buffered HF dip and 2 minute DI rinse, to remove native oxides from the anchor contacts. Particular care must be taken, because of the long HF release step which will follow. Doping and grain size in the polysilicon film may be chosen to suit; undoped, coarse-grained $\left(605^{\circ} \mathrm{C}, 100 \mathrm{~nm}\right.$ roughness) material was mainly used, since this gave the highest growth rate. Undoped material should be isolated from PSG to prevent doping-induced stress gradients. Films were annealed in nitrogen at $1000^{\circ} \mathrm{C}$ for 1 hour to remove residual stress. A thick film - e.g. 4-5 $\mu \mathrm{m}-$ is desired, to avoid damage during release and bonding. The film should fill the trenches defined for the stiffening ribs. A test structure consisting of a long cantilevered beam should be included for thickness measurement, since optical methods fail beyond $\sim 1 \mu \mathrm{m}$.

Polysilicon is defined by plasma etch. Again, photoresist step coverage is vital, to avoid "bumthrough" at the edges of the cavities. Tethers were designed to be robust, to prevent structures from detaching during the release. $4 \mu \mathrm{m}$ wide $\times 10 \mu \mathrm{m}$ long, and $30 \mu \mathrm{m}$ wide $\times 30 \mu \mathrm{m}$ long designs both worked satisfactorily. Eight anchors were provided for each $500 \mu \mathrm{m}$ square package. Flanges on the packages were $100 \mu \mathrm{m}$ wide. Narrower designs may be possible.

Gold deposition is preceded by a BHF dip, as above, to ensure adhesion in the release. In addition, $100 \mathrm{~nm}$ of chrome was deposited deposited as an adhesion layer. This also seems to prevent excessive diffusion of the gold during bonding, and the layer is apparently not undercut in the HF release. An evaporated gold film of $0.7-1.0 \mu \mathrm{m}$ thickness yields good results in bonding. Problems were encountered with films in the $100 \mathrm{~nm}$ range.

Following metallization, wafers were diced and released. Release required 25 minutes in concentrated (49\%) VLSI-grade HF. A surfactant (Triton X-100) was added to prevent collapse due to surface tension. A 5 minute DI rinse was followed by critical point drying in $\mathrm{CO}_{2}$ [17].

Target substrates consisted of $2 \mu \mathrm{m}$ thick polysilicon on a single-crystal substrate. Roughness was $10 \mathrm{~nm}$ peak-to-peak. Targets were prepared with the BHF dip procedure, followed by blow-dry with nitrogen. Handle and target chips were then contacted in a Class 100 environment. Contact was performed by laying one edge of the handle chip down on the target chip, then allowing the handle chip to fall flat. The handle could then be positioned laterally to align the two chips. The chips were placed in the bonding apparatus and clamped. The target chips were diced about $10 \%$ larger than the handle chips to simplify handling of the sandwich.

Approximately $60 \mathrm{kPa}$ of contact pressure was applied to the chips in the apparatus, shown in Figure 2. Note that this force is distributed only over the bonded areas, making the actual contact pressure about 1.2 $\mathrm{MPa}$. Dummy structures were placed at each comer of the layout to ensure parallelism of the chip faces.

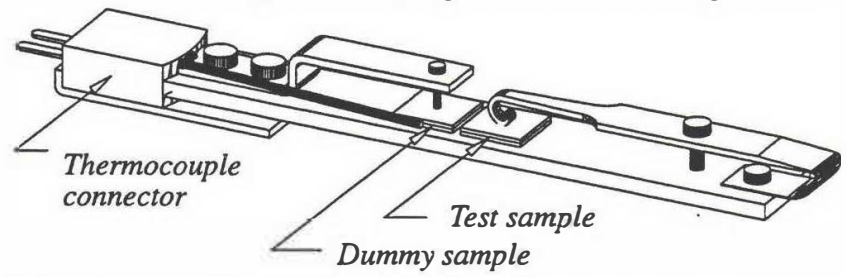

FIGURE 2. Bonding apparatus. The apparatus is placed in a quartz vacuum chamber and heated from below with an external lamp The clampling spring (right-hand side) applies contact pressure to the test sample.

Bonding was performed in a vacuum of $\sim 10^{-5} \mathrm{~T}$. The anodized aluminum surface of the bonding apparatus provides a susceptor, allowing consistent heating by the external lamp. A temperature of $370^{\circ} \mathrm{C}$ was found to be effective for Au/Si eutectic bonding. Temperature was monitored by a thermocouple mounted inside a sandwich of two silicon chips, clamped adjacent to the sample (see Figure 2). The thermal ramp-up to $370^{\circ} \mathrm{C}$ required approximately 5 minutes, after which cooling was begun immediately. Approximately 30 - 60 seconds was spent above the eutectic temperature. After cooling, the two chips were carefully separated to avoid shearing the microstructures. Separation breaks the polysilicon tethers (see Figure 1), leaving the shells bonded to the target. Results are shown in Figure 3.

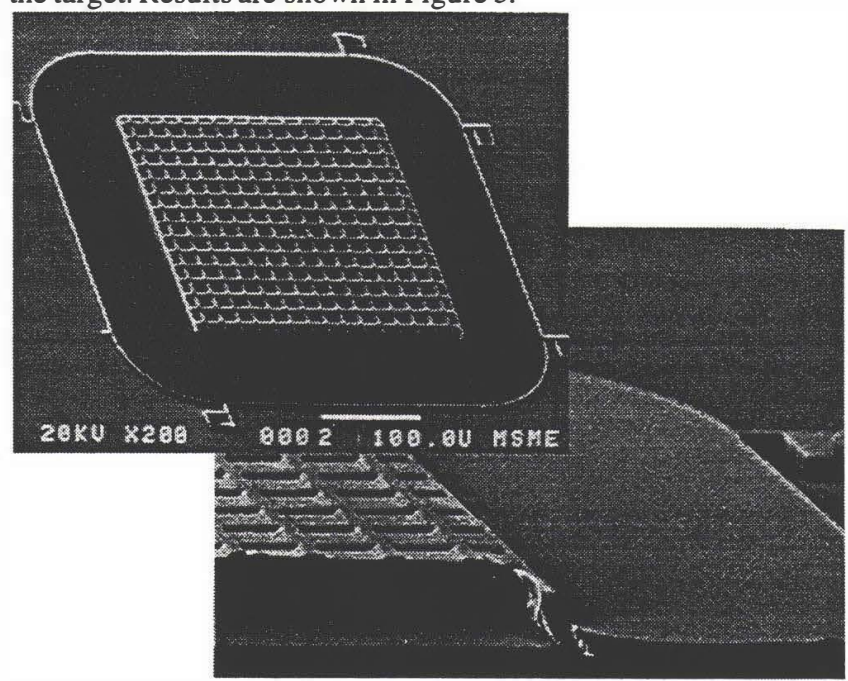

FIGURE 3. SEM of vacuum cap after transfer: Above: Some tethers are still partially attached to edge of cap. Flange (surrounding ribbed square) is $100 \mu \mathrm{m}$ wide. Below: Cleaved cross-section of ribbed cap.

Most of the packages were designed with ribs, so that there would be minimal deformation under atmospheric pressure. However, several packages were designed without ribs so that Nomarski imaging (Figure 4) could reveal a displacement of the shell. Profilometry indicated a displacement of up to $680 \mathrm{~nm}$ on $300 \mu \mathrm{m}$ square, $4.47 \mu \mathrm{m}$ thick structures. The displacement disappeared when the package was punctured. Substantial vacuum persists at the end of a five-month test, as shown in Figure 5.

Profilometer measurements after transfer indicate significant compression of the seal structure. From an initial thickness of 730 $\mathrm{nm}$, the gold layer is compressed to an apparent thickness of 346 $\mathrm{nm}$. Some of this compliance may have occurred in the adjacent 


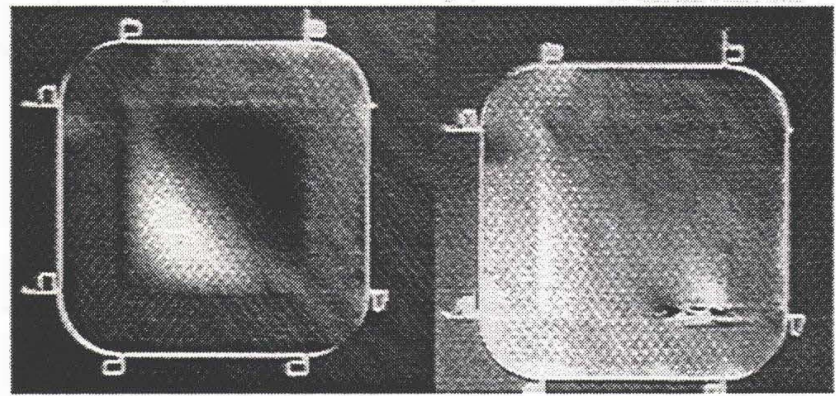

FIGURE 4. Cap without ribs was designed to deflect under pressure differential. Left: after assembly and anneal. Right: same, after being punctured. Nomarski imaging.

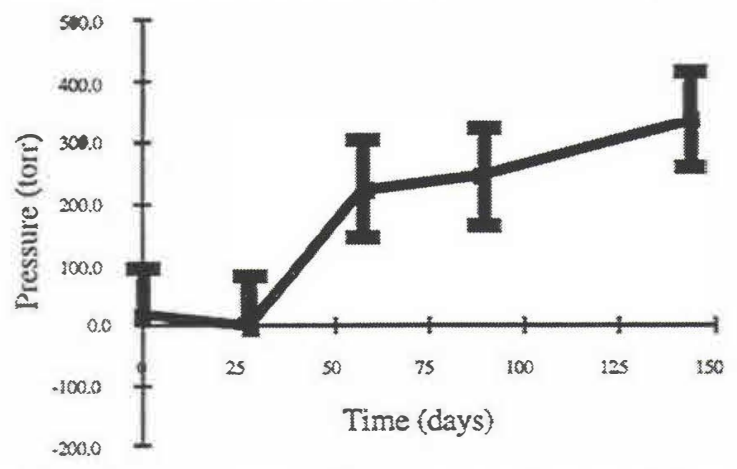

FIGURE 5. Package internal pressure as a function of time. layers of the structure (refer to Figure 1).

\section{DISCUSSION}

The transfer and bonding process described here displays several important fearires and unexpected advantages. Since a high degree of roughness is tolerated on the mating surfacer. packages can be pumped out after being assembled. This simplifies the apparatus considerably. In addition, the compression of the seal - more than $500 \mathrm{~nm}$ in some cases - suggests application to feedthrougins and other planarization problems.

There is evidence that complex, high-aspect structures can be transfered. The sample in Figure 6 was prepared by a "wet ransfer" process, in which release occurs after the two chips are contacted. Residual $\mathrm{SiO}_{2}$ from the $\mathrm{DI}$ rinse most likely causes bonding [18]. Subsequent processing included sputter deposition of $100 \mathrm{~A}$ of gold.

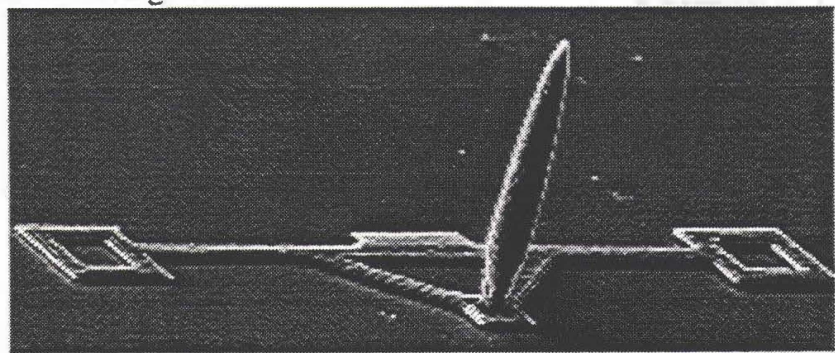

FIGURE 6. SEM of HexSil structure placed on SCS substrate by "wet rransfer" method. The vertical feature is 80 um in height.

Although these transfer processes appear robust to surace roughness and a modicum of particulate contamination, released structures are generally fragile and are potential particle generators themselves, if accidentally detached from the substrate. Fortunately, robust anchors can be liberally distributed, because of the high strength of the eutectic bond.

Shear strength of the bond was measured by bonding two unpatterned silicon chips. The resulting sandwich was supporte along two opposite edges and a load applied at the middle. A crack formed and propagated through the sandwich, with no evidence of de-bonding. A value of at least $0.39 \mathrm{GPa}$ may be calculated for the shear strength in this case. This is given by the shear stress at the neutral axis of the two-chip sandwich, when the maximal stress reaches the yield strength of silicon. The value seems reasonable in light of Lee's result of $5.5 \mathrm{GPa}$ for the tensile strength, achieved under similar annealing conditions [16].

To ensure survival of the caps curing release and contact, it may also be useful to avoid stress concentrators in the design. For example, hexagonal ribbing is preferred to square, and rounded corners to right angles.

A process has been developed for patterning the shells with reinforcement ribs. In fabrication, the sib structures are etched first, followed by the package cavities. In this way, the mask stepper need only focus at the plane of the wafer surface. Trench widths should be chosen as with HexSil [5]. Oxide thickness was minimized, on the premise that a thin sacrificial layer would prevent damage during contact, by avoiding excessive strain the anchors. Thicker layers may be usable.

Patterning of the sacrificial oxide is complicated by the topography. Poor resist coverage tends to occur at the edges of the cavities, leading to loss of the oxide and failure to release. Softening of these steps with an isotropic etch may be beneficial. Generous use of photoresist is recommended, e.g. $4 \mathrm{um}$ or more, depending on step height. Alternately, the cavity depth may be reduced, since sagging of the ribbed packages is negligible.

Some latitude is possible in choice of structural material. Both uncoped and doped, coarse- and fine-grained polysilicon recipes have been used. Silicon nitrice has boen shown by Lin [14] in a similar application, and gold is also a possibility.

Gold adhesion in the release step was variable. The preceding BHF dip seems vital, however. In one trial, a shor plasma etch $\left(\mathrm{CH}_{3} \mathrm{~F} / \mathrm{CF}_{4} / \mathrm{He}\right)$ was used instead, with the intent to maintain a dehydrated suface for the gold evaporation. Poor adhesion resulted in this case.

Accurate temperanure conrol is recommended. Significantly exceeding the eutectic temperature causes distortion of the polysilicon structures, apparently due to consumption by the liquid eutectic. An example is shown in Figure 7. Control of temperature and the initial gold distribution may lead to novel capabilities, such as plasticly deformed shells, welded links, or fusible supports.

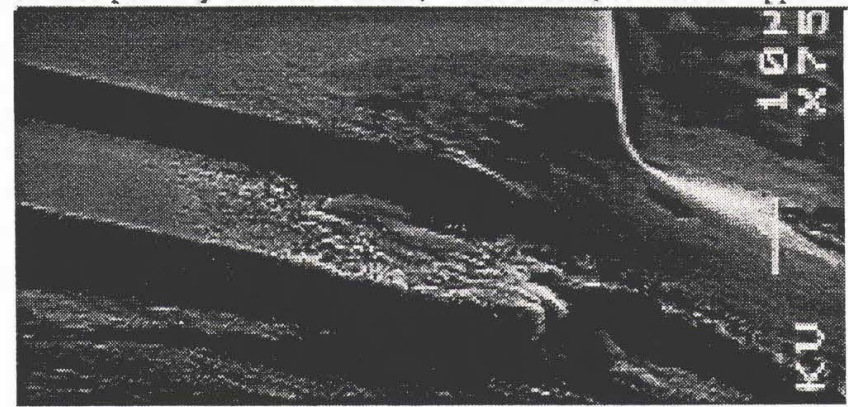

FIGURE 7. SEM of a comer of a vacuum package, after bonding on a $700 \mathrm{~nm}$ Au fim at $-400^{\circ} \mathrm{C}$. Note "smearing" and grainy appearance of material.

In the sealed structures, the differential pressure across the cap may be determined from the expression for plate deformation under a distributed load [19]:

$$
\Delta z=\frac{0.00126 q a^{4}}{D}
$$


where

$$
D=\frac{E h^{3}}{12\left(1-v^{2}\right)},
$$

the Poisson ratio of polysilicon, $v$, is taken as 0.23 , the Young's modulus, $E$, is $170 \mathrm{GPa}, \Delta z$ is the measured deformation of the top surface of the cap, the width of the plate, $a$, is $300 \mu \mathrm{m}$, and the thickness of the plate, $h$, is $4.47 \mu \mathrm{m}$. Rearranging, we find $q=$ $816 \pm 75 \mathrm{~T}$. The uncertainty is contributed mainly by $E$.

Since the ambient pressure at the time of measurement was - $752 \mathrm{~T}$, the internal pressure is expected to be in the range of 0 12 torr. The variation of this value with time is plotted in Figure 5. The long time constant suggests outgassing rather than leakage; a more aggressive bakeout, or gettering, might yield improvement.

\section{WORK IN PROGRESS}

Alignment of handle and target was achieved by patterning a $1.7 \mu \mathrm{m}$ oxide step on the target, contacting the two chips, and abutting features on the handle against the step. A sharp piece of silicon was dragged across the back of the handle chip to generate a lateral force. It was expected that this force would be limited by the $\mathrm{Si}-\mathrm{Si}$ friction coefficient, and thus would be commensurate with the forces present on the front side of the chip. Misalignment after bonding was less than the detection limit of $5 \mu \mathrm{m}$.

Encapsulated resonator structures were designed using a periodic pattern of conductive feedthroughs to favor planarization [12]. A $300 \mathrm{~nm}$ doped polysilicon film was used in a $5 \mu \mathrm{m}$ linespace pattern, shown in Figure 8. A $2 \mu \mathrm{m}$ LTO/PSG layer was overlaid for planarization, followed by $1 \mu \mathrm{m}$ of low stress silicon nitride to protect it from the release. The same oxide film was also used for the resonator sacrificial layer. $2 \mu \mathrm{m}$ polysilicon doubles as the resonator structural layer and a buffer layer over the feedthroughs for additional planarization. This surface displayed a $170 \mathrm{~nm}$ height modulation due to the feedthroughs, suggesting that a seal should be readily achieved, considering the $500 \mathrm{~nm}$ compliance described above. The die also includes multi-bore needles with internal electrical feedthroughs, hinge structures, and transferred resonators.

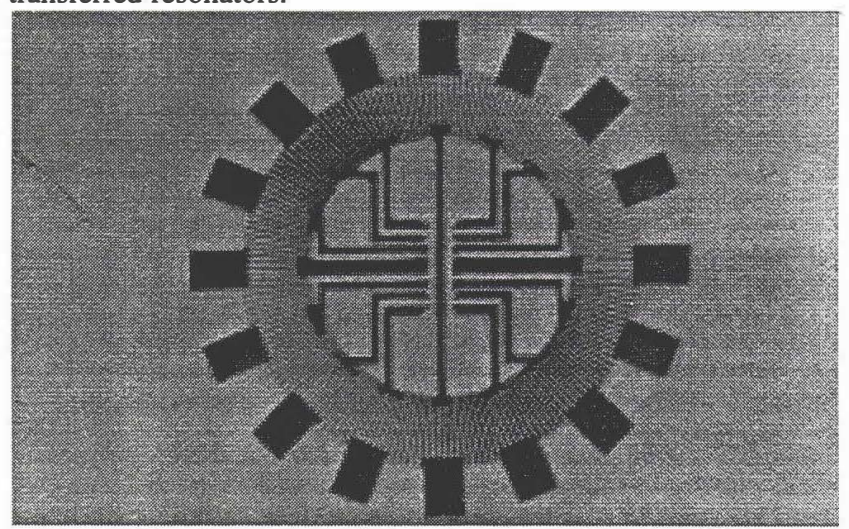

FIGURE 8. Feedthrough structure for encapsulated resonator. 3000A doped polysilicon connections are supplied for an electrostatic comb drive, sensing capacitors, and fusible supports.

Subsequent work will pursue both improved vacuum packaging and more general applications. For instance, the transfer process may be proposed for sensor structures, i.e. to combine resonators with CMOS. GaAs devices are also a candidate. This approach avoids many process-compatibility problems, and offers to simplify MEMS development: prefabricated, VLSI-scale modules (op-amps, microcontrollers) could be incorporated into many systems by assembly, rather than monolithic integration.

\section{CONCLUSION}

A technique has been demonstrated for batch transfer of complex polysilicon structures between two wafers. A gold/silicon eutectic forms a high-strength bond and hermetic seals. The bonding process is robust to surface roughness and contamination, and can be performed at low temperature. Packages can be evacuated after assembly, simplifying equipment design.

\section{ACKNOWLEDGMENTS}

The authors thank Mark Noworolski for sharing his results on gold adhesion. This work was funded by ARPA under contracts FA-ADI-DTD8/5/93 and DABT63-95-C-0028, and by the Berkeley Sensor \& Actuator Center.

\section{REFERENCES}

1. Roessig, T., Pisano, A. P., and Howe, R., T., "Surface Micromachined Force Sensor," Proceedings of the ASME Dynamic Systems and Control Division. ASME, 1995, p. 871

2. Bemstein, J.; Cho, S.; King, A.T.; Kourepenis, A.; and others, "A micromachined comb-drive tuning fork rate gyroscope," Proceedings, IEEE Micro Electro Mechanical Systems., Fort Lauderdale, FL, 7-10 Feb. 1993, p. 143-8.

3. Clark, W. A., "Surface Micromachined Z-Axis Vibratory Rate Gyroscope," Technical Digest of the 1996 Solid-State Sensor and Actuator Workshop, Hilton Head, SC, 6/2-6/96

4. Juneau, T. and Pisano, A. P., "Micromachined Dual Input Axis Angular Rate Sensor," Technical Digest of the 1996 Solid-State Sensor and Actuator Workshop, Hilton Head, SC, 6/2-6/96.

5. C. G. Keller and M. Ferrari, "Milli-Scale Polysilicon Structures," Technical Digest of the 1994 Solid-State Sensor and Actuator Workshop, Hilton Head, SC, 6/ 13-16/94

6. Wise, K. D., Robinson, M. G., and Hillegas, W. J., "Solid-State Processes to Produce Hemispherical Components for Inertial Fusion Targets," J. Vac. Sci. Technol., 18, April 1981, p. 1179-1182.

7. Petersen, K., Barth, P., Poydock, J., and others, "Silicon Fusion Bonding for Pressure Sensors,” 1988 Solid-State Sensor and Actuator Workshop, Hilton Head, SC, 6/6-9/88, p144-7.

8. Huff, M. A., Nikolich, A. D., and Schmidt, M. A., "A Threshold Pressure Switch Utilizing Plastic Deformation of Silicon," 1991 International Conference on Solid-State Sensors and Actuators, IEEE, San Francisco, CA, 6/24-27/91, p.177.

9. Ikeda, K., Kuwayama, H., et al, "Three-dimensional Micromachining of Silicon Pressure Sensor Integrating Resonant Strain Gauge on Diaphragm," Sensors and Actuators, A21-23, 1990, p. 1007-10.

10. Sniegowski, J.J.; Guckel, H.; Christenson, T.R. "Performance characteristics of second generation polysilicon resonating beam force transducers." IEEE SolidState Sensor and Actuator Workshop Hilton Head Island, SC, 6/4-7/90 p. 9-12.

11. Hoogerwerf, A. C. and Wise, K. D., "A Three-Dimensional Neural Recording Array," 1991 International Conference on Solid-State Sensors and Actuators, IEEE, San Francisco, CA, 6/24-27/91, p. 120.

12. Ziaie, B., Gianchandani, Y, and Najaf, K., "A High-Current IrOx Thin-Film Neuromuscular Microstimulator," 1991 International Conference on Solid-State Sensors and Actuators, IEEE, San Francisco, CA, 6/24-27/91, p. 124

13. Mastrangelo, C. H., Muller, R. S., Kumar, S., "Microfabricated Incandescent Lamps," Applied Optics, 1 March 1991, vol.30, (no.7):868-73.

14. Lin, L., McNair, K. M., Howe, R. T., and Pisano, A. P., "Vacuum Encapsulated Lateral Microresonators," 1993 International Conference on Solid-State Sensors and Actuators, IEEE, p. 270-3.

15. Ko, W. H., Suminto, J. T., and Yeh, G. J., "Bonding Techniques for Microsensors," Micromachining and Micropackaging of Transducers, Elsevier, 1985, p. 41. 16. Lee, A. P., Ciarlo, D. R., and others, "A Practical Microgripper by Fine Alignment, Eutectic Bonding, and SMA Actuation," Digest of the 1995 Intermational Conference on Solid-State Sensors and Actuators, Stockholm, Sweden, 6/25-29/ 95, p. 368-71

17. Mulhem, G. T., Howe, R. T., and Soane, D. S., "Supercritical Carbon Dioxide Drying of Microstructures,"1993 International Conference on Solid-State Sensors and Actuators, IEEE, p. 296-9.

18. Judy, M. W., Ph.D. Thesis, EECS Department, University of California, Berkeley, 1994, p. 46 ff.

19. Roark, R. J., and Young, W. C., Formulas for Stress and Strain, McGraw-Hill, 1982.]

20. Minami, K., Moriuchi, T., and Esashi, M., "Cavity Pressure Control for Critical Damping of Packaged Micro Mechanical Devices," Transducers '95, 1995, Stockholm, Sweden.

21. Monk, D. J., Krulevitch, P., Howe, R. T., and Johnson, "Stress-Corrosion Cracking and Blistering of Thin Polycrystalline Silicon Films in Hydrofluoric Acid," Symposium on Thin Films: Stresses and Mechanical Properties IV, MRS Spring Meeting, San Francisco, CA, 5/93. 